\title{
Comparative Study of Circulate Stabilizing Vs Circulate Retaining Prosthesis in Total Knee Replacement
}

\author{
Mohan Choudhary.B ${ }^{1}$, Deepak Kumar Srinivasan ${ }^{2}$, Mohan Kumar $\mathbf{M}^{3}$ \\ (Department of Orthopedics Sri Ramachandra Medical College / University, India)
}

\begin{abstract}
A retrospective analysis was carried out to compare cruciate-retaining $(C R)$ and cruciate-stabilized (CS) total knee replacements (TKRs). A total of 30 knees were analyzed which received CR or a CS, TKR. All knees were implanted with identical surgical technique, making sure to balance precisely the flexion-extension gaps before implantation of the components. At 1 year follow-up, there was no difference between the CR and the CS TKRs with respect to their Knee Society clinical and functional score. These findings suggest that with careful attention to surgical technique and balancing the knee, orthopedic surgeons should expect similar results whether they use a CR or CS TKRs.
\end{abstract}

Keywords: $C S, C R, T K R$

\section{Introduction}

The short term results of cruciate retaining (CR) and cruciate stabilized (CS) total knee replacements (TKRs) have shown their ability to relieve pain and improve function. Nevertheless, the controversy continues regarding the superiority of one design type over the other. Proponents of CR TKR believe that maintaining the posterior cruciate ligament (PCL) provides increased stability and promotes femoral rollback, enhancing stairclimbing ability. The maintained PCL can absorb shear forces that otherwise would be transmitted to the boneimplant interface and could result in premature loosening. Proponents of CS TKR argue that resection of the PCL facilitates correction of deformity and allows for the use of more congruent articular surfaces, minimizing polyethylene wear.

Although some studies found no difference between CR and CS TKR, others showed improved range of motion with PCL resection. These studies failed to take into account that differences between CR and CS TKRs may be related to implant design or surgical technique or both. The purpose of the study was to analyze the clinical and functional results using knee society scoring system in patients undergoing CR and CS TKR.

\section{Aim}

To compare the clinical and functional outcome between cruciate stabilizing and cruciate retaining prosthesis following total knee replacement

\section{Materials And Methods}

A total of 30 knees were analyzed which received either a CR TKR or a CS TKR knee. It is a retrospective analysis. The study period was from Dec 2009 to Dec 2012.

No patients were lost to follow-up. All knees were followed for 6 months to a minimum of 1 year postoperatively. 16 knees were followed up for more than 4 yrs. CR TKR was implanted in 17 knees, whereas CS TKR was implanted in 13 knees. TKR was done for osteoarthritis in 26 knees. The diagnosis was rheumatoid arthritis in 2 knees in the CR group and 2 knees in the CS group. The mean age of the patients at the time of surgery was 60 years in the CR group and 65 years in the CS group. At the time of surgery, the average weight of the patient in the CR group was $70 \mathrm{Kgs}$ and $74 \mathrm{Kgs}$ in the CS group. The average height was $155 \mathrm{cms}$ in the CR group and $160 \mathrm{cms}$ in the CS group. Knees with more than 20 degrees of varus or 20 degrees of FFD usually received CS knee.

All surgeries were done by the senior consultant. The surgical technique involved approaching the knee through a midline incision and a medial Para patellar arthrotomy. All varus-valgus deformities were corrected using appropriate soft tissue releases. The instrumentation was used to obtain accurate rotation of the femoral component along the epicondylar axis. After sizing the anteroposterior dimensions of the distal femur, the distal femoral cutting guide was aligned along the trans epicondylar axis, and the distal femur was cut. A vertical slot was made perpendicular to the transepicondylar axis on the cut femur to maintain the desired rotation for the anterior-posterior resection guide. The anterior posterior cutting block was adjusted to cut $9 \mathrm{~mm}$ inferiorly. If the knee was planned for a CS TKR, the anterior cruciate ligament and PCL were resected. The proximal tibia was addressed using extramedullary tibial cutting guide; $10 \mathrm{~mm}$ of bone was resected off the proximal tibia from the least affected side. The flexion-extension gaps were measured using spacer blocks. If necessary, further femoral or tibial cuts were carried out to balance the flexion-extension gap. When the 
flexion-extension gaps were balanced, the distal femur was finished. The anterior and posterior chamfer cuts were made, as were the distal drill holes for the pegs. In the case of the CS TKR, an intercondylar notch cut was made. The proximal tibia was prepared to receive the tibial base plate, which was identical in both groups. In neither cases patella was resurfaced. All components (PFC (Depuy) or TC PLUS (PLUS ORTHO)) were cemented in place using hand-mixed cement.

Postoperatively, all patients were allowed full weight bearing as tolerated. Physiotherapy and continuous passive motion were started on all patients on postoperative day 1 , and physiotherapy was continued for 6 weeks postoperatively. Patients were discharged after 10 days following suture removal and achieved a minimum of 90 degrees flexion. Preoperatively, all patients were assessed with regard to diagnosis, gender, age, height, and weight. All patients were evaluated preoperatively and at 6 weeks, 3 months, 6 months and 1 year postoperatively. The knee Society clinical scoring systems were used to evaluate the patients. The preoperative radiographs were compared. The prosthesis bone interface was evaluated for the presence and progression of radiolucent lines. Progression of a radiolucent line was defined as an increase in length or width or both of 2 $\mathrm{mm}$ on sequential radiographs. A change in knee or prosthetic alignment was considered significant when it was 3 degrees.

\section{Results}

There was no difference between the CR and CS TKRs with respect to their preoperative and 1 year postoperative Knee Society clinical and functional scores. There were no intraoperative complications in either group, and no knees have required revision surgery in this short follow-up. Preoperatively the Knee Society clinical score averaged 35 in the CR group and 37 in the CS group. At 1 year follow-up, the clinical score improved to a mean of 100 (range 90-110) in CR group and 95(range 90-100) in CS group. There was no difference in the range of motion between the CR and CS TKRs preoperatively or at any time postoperatively. Preoperatively the mean flexion contracture was $12^{\circ}$ in the CR group and $20^{\circ}$ in the CS group. Knee flexion averaged $80^{\circ}$ in the CR group and $90^{\circ}$ in the CS group. The mean flexion at 1 year follow-up was $110^{\circ}$ in the CR group and $120^{\circ}$ in the CS group. Patients in both groups had a dramatic improvement in pain relief 3 weeks after TKR. 4 patients in the CR group and 3 patients in the CS group had mild occasional pain. There was no difference between the CR and CS TKRs with respect to the Knee Society functional score preoperatively or at 6 month to 1 year follow-up. Preoperatively the functional score averaged 34 in the CR group and 37 in the CS group. At final 1 year follow-up, the mean functional score was 95 in the CR group and 90 in the CS group. 10 knees in the CR group and 8 knees in the CS group were able to walk an unlimited distance. 1 patient in CS group was housebound because of significant medical problems (Rheumatoid arthritis) unrelated to their knee. Stairs were climbed normally up and down in 15 knees in the CR group and 8 knees in the CS group. Ambulation required no support in almost all CR knees and 2 knees in the CS group (Rheumatoid arthritis) required a cane for either long walks or for regular walking.

\section{Discussion}

This study shows that with identical surgical technique, there is no difference in the results of CR or CS TKR. The use of identical surgical technique in the CR and CS groups allowed us to isolate the effect of implant design on outcome after TKR. With careful balancing of the flexion-extension gaps intraoperatively, there is no difference between CR and CS TKRs with respect to functional outcome, range of motion, at 6 months to 1 year follow-up.

It is well accepted that accurate bony cuts, restoration of the mechanical axis of weight bearing, and soft tissue balancing are fundamental in achieving a successful TKR. The concept of equalizing the tibiofemoral space in $90^{\circ}$ of flexion and at full extension initially was introduced as a way to stabilize knee function when the PCL was resected. The concept of balancing the flexion extension gap is adhered to rigorously by all surgeons who do a TKR with a CS implant. Many CR implant systems do not allow for the measurement of flexion-extension gaps, and many surgeons assume that the gaps are balanced after the standard cuts. Inequalities in the flexion extension gaps in CR knees sometimes are corrected with soft tissue releases rather than bony cuts. As a result, CR and PS TKRs are different in implant design and surgical technique. Studies assessing the difference between these implant designs must take into account the surgical technique. In this study, the surgical technique was identical between the 2 implant designs, eliminating this variable.

Few studies have directly compared the effect of PCL retention or resection on outcome after TKR. Hirsch, Lotke, and Morrison reported on 242 consecutive, primary TKRs that were included in 1 of 3 sequential groups. They compared 77 press Fit Condylar TKRs in which the PCL was completely released, 80 press fit condylar TKRs in which PCL was retained and 85 TKRs with a PCL-substituting implant (Install-Burstein II; Zimmer, Warsaw, IN). At 2.6 to 2.7 years postoperatively, the PCL-substituting group had significantly greater range of motion than either the PCL sacrificed or retained group. There was no difference in the range of motion between the PCL sacrificed and retained TKRs suggesting to the authors that the PCL does not function as 
expected when it is retained after TKR. There were no differences among the 3 groups with respect to Knee Society clinical, function, or radiographic scores.

Maloney and Schurman reported on 51 knees that underwent TKR with Total Condylar prosthesis (Johnson \& Johnson, Braintree, MA) versus 53 TKRs with a CS Total Condylar implant. At 1-year follow-up, there was no statistical difference between the 2 groups with respect to preoperative or postoperative function scores, pain scores, or ability to climb stairs. The CS group had significantly better postoperative flexion compared with the Total Condylar group; however, the CS group also had significantly better preoperative flexion.

Dorr and Ochsner et al reported a functional comparison of CR versus cruciate-sacrificing TKR. Sixteen patients who underwent bilateral TKR received a posterior cruciate-sacrificed TKR (Total Condylar) in 1 knee and 1 of 3 different CR implants in the contra lateral knee. Five patients were eliminated from the study because they developed other joint symptoms. At 2 years postoperatively, gait laboratory data analysis on the remaining 11 patients revealed that the posterior cruciate-sacrificed TKR was less efficient, had greater medial loading, and higher joint reaction forces. The authors concluded these gait abnormalities may affect the durability of posterior cruciate-sacrificed TKRs. The 5-year Hospital for Special Surgery knee scores, patient satisfaction, and radiographic examination were the same for both implants.

In another bilateral TKR study, Becker, Insall and Faris reported on the 2-to 5-years follow up of 30 patients who underwent bilateral TKR. One knee received an Insall-Burstein (Zimmer, Warsaw, IN) CS prosthesis, whereas the contra lateral knee received 1 of 3 different CR prostheses. In general, the more deformed knee received the CS implant. Laskin showed a poorer outcome in patients undergoing TKR with a CR design if they have significant preoperative flexion and varus deformities. Becker, Insall, and Faris found no difference between the 2 groups with respect to Hospital for Special Surgery knee score, range of motion, stair-climbing ability, or patient satisfaction. The authors concluded that there is no clinical advantage of one type of TKR over the other. Udomkiat and Meng et al conducted a matched-pair analysis to compare CR and CS TKRs. Of 150 consecutive TKRs, 38 matched pairs were included for this study. Patients received either a CR or CS Apollo TKR. With the exception of a traditional posterior stabilizer housing and 2 fixation pegs, the Apollo CS femoral component was identical to the CR component. At 2-year follow-up, the authors found no difference in the Knee Society patient functional scores or range of motion between the 2 implant designs. The authors concluded that surgeons who prefer to resect the PCL should expect a clinical result as good as surgeons who retain the PCL. These Fluoroscopic analyses of TKRs showed that although CS knees showed posterior femoral rollback, dictated by the interaction of the femoral cam and tibial post mechanism, there was a large variability in contact pathways between varying CS implants. The implants used in the current study more closely represent the current philosophy of orthopaedic surgeons, who have a preference of one implant design over the other. Aside from retaining or substituting for the PCL, all knees were implanted with the identical surgical technique.

\section{Conclusion}

This study suggests that with careful attention to surgical technique and balancing the knee, orthopaedic surgeons should expect similar results whether they use a CR or CS TKR. When the flexionextension gaps were balanced accurately, we could find no difference in the clinical and functional outcome of CR or CS TKRs at 1 year postoperatively in this short term analysis.

\section{References}

[1]. Becker MW, Insall JN, Faris PM: Bilateral total knee arthroplasty. Clin Orthop 271: 122, 1991.

[2]. Dorr LD, Ochsner JL, Gronley J, Perry J: Functional comparison of posterior cruciate retained versus sacrificed total knee arthroplasty. Clin Orthrop 236:36, 1988.

[3]. Hirsch HS, Lotke PA, Morrison LD: The posterior cruciate ligament in total knee surgery. Clin Orthop 309: 64, 1994.

[4]. Maloney WJ, Schurman EJ: the effects of implant design on range of motion after total knee arthroplasty. Clin Orthop 278:147, 1992.

[5]. Schai PA, Thornhill TS, Scott RD: Total knee arthroplasty with the PFC system: Results at a minimum of ten years and survivorship analysis. J Bone Joint Surg Br 50:850, 1998.

[6]. Scott RD, volatile TB: Twelve year experience with cruciate-retaining total knee arthroplasty. Clin Orthop 205: $100,1986$.

[7]. Udomkiat P, Meng B4J, Dorr LD, Wan Z: Functional comparison of posterior cruciate retaining and substitution knee replacement. Clin Orthop 378: 192, 2000. 